\title{
College Students’ Sports Consumption and College Physical Education in China
}

\author{
Ding Yi \\ Pingdingshan Institute of Education, Pingdingshan Henan China, 467000
}

\begin{abstract}
The sports consumption trend of college students has some instructive significance for higher physical education. Chinese College students' sports consumption has its specific content, motive and levels, it reflects some background factors such as economic, class and culture and different developmental needs for physical development of college students. College physical education should meet the demands of different students in different levels and situations. In the concrete teaching process, college physical education need to strengthen the teaching of richness, targeted, flexibility and education guidance. Measures such as consolidating foundation teaching, increasing the depth of the teaching, exploring the layered teaching and strengthening the guided teaching should be taken to make full use of the utility of sports resources as well as enlarging basic physical education's coverage.
\end{abstract}

Key words: college; students' sports consumption; physical education in college

\section{Consumption's Enlightenment for Edu- cation}

Consumption behavior is happening everywhere in society nowadays. During the process, people meet their life needs as well as psychological needs. Consumption behaviors reflect consumers' psychological preferences as well as social status and behavior tendency. As Baudrillard announced in Consumer Society, consumption is more than material practices or the feelings and experience on food, clothes, car, images and information, but a systematic semiotic manipulation that structuralizes referent (the real thing) into signifiers (the marker for the concept of the real thing). ${ }^{1}$

In a sense consumption is marked by social identities. The consumer behaviors are quite explicit to be translated and understood. Generally consumption is described as a re-coding process during which people define and understand the value and symbol of a product, and then build self-identity with these signs. Through divergent consumption, people set their boundaries against outside or other social groups, establishing the symbols and marks of their own social status.

Resembling a compass, consumption shows certain groups' taste, attitude and behavior tendency. College student is a distinct social group with good knowledge and qualities. Their self-identity is formed and maintained through specific consuming behaviors and habits, which in turn, expose their basic needs and development prospect. Hence understanding of their sports consumption behaviors would greatly assist future college education reform and development. Additionally analyzing students' consuming features and trends would also help academic teaching in colleges, which has some instructive significance for higher physical education.

\section{The Objects and Features of Sports Con- sumption among Chinese College Student}

Socialist Kell states that individual's consuming feature is usually influenced by social class but income. On one hand, social structure "constrains" people's choice on consuming mode. To be more specific, the way "I" purchase shall to fit "my" social status. On the other hand, modern consumption is capable to dissolute and restructures social class, i.e. our consuming objects and pattern are telling who we are or who we want to be. This is how modern consumption sociology demonstrates the connection between modern consumption and social structure. ${ }^{2}$ Chinese college students, as a special group, are trying to construct social identity. In a word, despite the differences of economic background, sports consumption group among college students is affected by its social group identity and bear certain group features.

\subsection{Consuming objectives}

To Chinese college students, their purchasing objectives mainly include sports consumables, participatory and ornamental activities. ${ }^{3}$ Consumables are products like apparels (sportswear, sneakers), equipments (sports machines and equipments) and groceries (energy drinks and foods). Participatory consumption refers to the expenses on sports activities, for example joining sports club and gym, renting sports venue. Ornamental consumption includes all the expenses on watching sports game and activities.

Comparing with other groups, college students are full of energy and creativity that they always ready to try new things. Although lacked of purchasing power, enthusiasm drives them to try more varieties of sports products. 


\subsection{Consumption Motive}

The environment, age and social character determine that college students have unique consumption motive. According to the survey, there are 5 types of motive behind sports consumption of college students.

\subsubsection{Fitness Motive}

Everyone desires physical health. College students are in their best age and key period of building up bodies. Most students, both male and female, agree to spend some money on fitness ${ }^{1}$, believing keeping regular exercises is good to health.

\subsubsection{Communicating motive}

Sports consumption, especially participatory activities, is regarded as a efficient way to expand social circle. Study shows that male students' communicating motive highly exceeds female students', for instance male students are more likely to take the initiative in communication with other people at gym.

\subsubsection{Aesthetic Motive}

Physical exercises help to build muscular physique and freshen the mind. A great number of students, especially female students, are insisting on building physique and body figure for better appearance and temperament.

\subsubsection{Semiotic Motive}

With its semiotic feature, high-end consumption, for example high class gym, brandy apparels, is seen as a symbol of good taste, high status, and strong financial capability. Many students from affluent families are fascinated with brandy products, showing off their wealth, family background and social class.

\subsubsection{Conformity}

Conformity is wildly existed. When one sport product is prevailing among college students, those who are lack of rational consuming habit would chase the trend blindly without considering their actual purchasing power. Conformity acts as an important driving force in sports consumption among small groups in college.

\subsection{Consumption Level}

All the analysis above is indicating that college students' sports consumption has clear levels: basic consumption, developing consumption and showing-off consumption. Basic consumption is triggered by the desire for fitness. Developing consumption is driven by communicating and aesthetic motive while showing-off expenses propelled by semiotic motive and conformity.

\footnotetext{
${ }^{1}$ Baudrillard, J., Liu C. F., Quan Z. G., (2000). The Consumer Society. Nanjing: Nanjing University.

2 Zuo X. R., (2004). Analysis on General and Developing Sports Consumption. Beijing: Beijing Sports University Journal.

${ }^{3}$ Zhang H., Luo J. Y., (2002). Investigation and Analysis on College Students Consumption Psychology and Behavior. Shandong: Shandong Sports College Journal.

${ }^{4}$ Huo D. L., Mao X. Y.(2008). The Study on the Characters of College Students Sports Consumption Psychology and Behavior. Shaanxi: Shaanxi Normal University Sports Department Journal.

${ }^{5}$ Zhang H., Luo J. Y., (2002). Investigation and Analysis on College Students Consumption Psychology and Behavior. Shandong: Shandong Sports College Journal.
}

\section{Reviewing Chinese College Education from Consumption Aspect}

Due to the unique feature and pattern of sports consumption of Chinese college students, be familiar with their tastes and preferences would facilitate the understanding of further needs of physical teaching and exercises in college.

In a consumption aspect, physical teaching, as analyzed above, should be further developed with more strict standards.

\subsection{Diverse Consumption and Academic Teaching}

Diversity is the most obvious feature of Chinese students' sports consumption. It varies from material consumption (sports facilities, products and apparels) to participatory consumption and ornamental consumption. However the fact is that today's teaching context in college is dominated by physical education class, which means participatory sports education occupies more teaching resources. Meanwhile other type of sports education, such as sports game, sports performance, is relatively ignored. In other words, the singular and tedious class is unable to meet students' diverse requirements for physical development.

\subsection{Consuming Preference and Teaching Pertinence}

The consuming preference varies from student to student, but generally males prefer strenuous exercise, like ball games and strength training while females favor soft and light sports that help building body figure, such as jogging, dance sport, yoga, etc. Consequently the academic teaching should consider increasing the types and pertinence of sports education on the basis of guaranteeing basic sports training. In addition, students' physical status, age, gender shall be considered when drawing up a training plan.

\subsection{Consumption Layers and Education Flexibility}

The sports consumption of Chinese college students could be divided into different levels. Basically students' preferences on sports products and activities are divergent due to diverse family background, culture, tradition and economic condition. To be more specific, students from lower class seldom spend money on extra sports consumption while those from urban areas are willing to pay large amount for gym and apparels. Considered the current situation, physical education shall be more flexible after ensuring the free fundamental education, for instance, paid class and sports venue could be introduced as extra choices for students.

\subsection{Semiotic Consumption and Education Orientation}

The semiotic consumption implies the behaviors of showing off wealth by purchasing brandy products or joining high class fitness club. This represents a phenomenon of modern consumption society. However excessive semiotic consumption violates the spirit of modern sports, hinders the promotion of sports spirit, and harms the goal of education. In college education, the 
significance of physical health education equals to psychological and mental guidance. During teaching process, it's crucial to explain sports spirit and meaning, state the norm and guide students to right direction. Only by doing so, will sports education comprehensively play its role.

\section{Reform Direction and Practical Meaning}

From the sports consumption viewpoint, Chinese sports course is relatively singular and lacks of pertinence and clear layers. Based on the analysis of consumption characters, motive and layers, improvements shall be adopted on the following aspects:

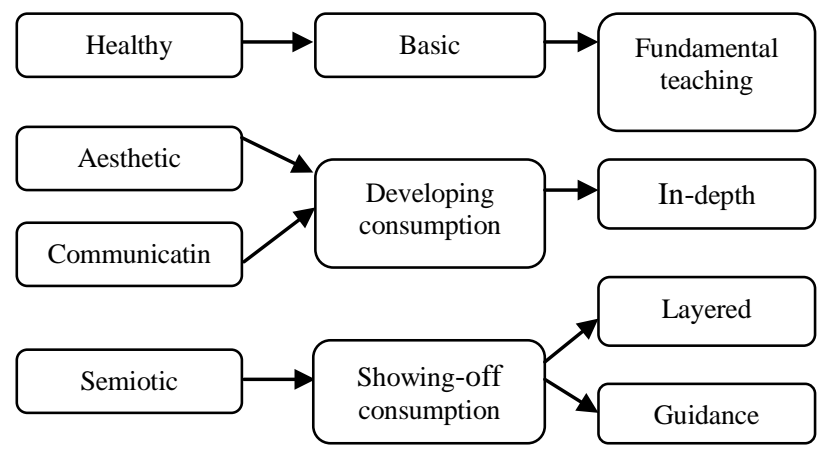

Figure 1. Guidance of College Students' Sports Consumption on Sports Education in College

\subsection{Enhance Fundamental Teaching}

As the root and basis of sports education, fundamental teaching is committed to enhance physique building and physical education development. College education has advantage of rich experiences and solid base, faculty resources and facilities resources in fundamental teaching, as a consequence, college authority should guarantee the quality and scale of fundamental teaching, meeting all students' requirements of development.

\subsection{Deepen Teaching Level}

As to the aesthetic and developing requirements, content of courses is the key to improvement. Distinction teaching method might be a useful approach as it emphasize on instructing different content to students of different gender and age.

\subsection{Exploring Layered Teaching}

Layered teaching is designed to meet diverse needs. After guaranteeing the normal operation of fundamental class, authority could utilize sports resources to diversify sports activities, for instance, open stadium and sports venue at different time section, thus granting more flexibility, at the same time, allowing public resources to be fully utilized.

\subsection{Emphasize Guidance Teaching}

Considered semiotic consumption is widely existed among students, college authority is obliged to take action to correct and guide students' behaviors through sports education. Adding classes about sports culture and spirit is a good way to teach students right concept about sports development and enable them to fully enjoy the spirit and emotional pleasure.

\section{Conclusion}

As members of a special social class, Chinese college students group bears its unique characters and preferences. Consequently, the college sports education needs to get rid of the traditional teaching constrains, deploying specific teaching methods that fit students' characters and real needs. Sports consumption of college students reflects their real requirements on sports; in a sense, it inspires the sports teaching reform and helps to locate the future direction. Only by continuing reform and innovation can sports education adapt constant changing society and requirements and play its crucial role in higher education mechanism.

\section{Reference}

[1] Baudrillard, J., Liu C. F., Quan Z. G., (2000). The Consumer Society. Nanjing: Nanjing University.

[2] Huo D. L., Mao X. Y.(2008). The Study on the Characters of College Students Sports Consumption Psychology and Behavior. Shaanxi: Shaanxi Normal University Sports Department Journal.

[3] Zuo X. R., (2004). Analysis on General and Developing Sports Consumption. Beijing: Beijing Sports University Journal.

[4] Zhang H., Luo J. Y., (2002). Investigation and Analysis on College Students Consumption Psychology and Behavior. Shandong: Shandong Sports College Journal. 